\title{
A MULTI-SENSORY APPROACH TO 3-D MAPPING OF UNDERGROUND UTILITIES
}

\author{
Leonhard E. Bernold, \\ Associate Professor \\ Lashminarayan Venkatesan, $G R A$ \\ Sushil Suvarna, GRA \\ Construction Automation Robotics Laboratory (CARL), North Carolina State University.
}

\begin{abstract}
The demand for new buried utilities, such as gas, power and fiber-optic communication lines is growing with new construction, re-construction, and the growth of the communication infrastructure worldwide. Because the machinery for placing the new utilities underground, such as backhoe excavators, trenchers, augers, drills, and plows, don't "feel" when they are getting close to already buried object, utilities are easily damaged. Despite great efforts in locating existing utilities before a contractor is allowed to dig, accidents occur. This paper will present a novel technology for detecting and locating buried utilities that attaches to the digging equipment and utilizes both EMI (Electromagnetic Induction) and a GPR (Ground Penetrating Radar). The newest effort involves the development and performance analysis of algorithms to detect and extract the features and characteristics of these utilities, such as their orientation, diameters etc. One prime focus is to minimize the percentage of false alarms. For that purpose, the two sensor systems are fused to create a multi-sensory approach to 3-D mapping of all the utilities without a priori knowledge of their location
\end{abstract}

Keywords: Buried utility detection, Electromagnetic Induction, Ground Penetrating Radar, Sensor fusion.

\section{INTRODUCTION}

In 1993, the Construction Automation and Robotics Laboratory (CARL) at North Carolina State University (NCSU) started an initiative to address the national problem of detecting and locating underground buried utilities. The core idea was to provide the equipment operator with his own system integrated with the equipment that alerts him of the danger rather then to depend on the color marks made by the locator sent by the One-Call center. For that purpose, a sensing platform, operating like a subsurface " $\mathrm{X}$ ray" was attached directly to the machinery hence providing the operator with an opportunity to "see" and be warned when the machine tool gets close to an existing utility. The original system was based on the Electro Magnetic Induction (EMI) technology that was integrated with PCbased software to process and analyze the signal coming from an antenna. Using a traditional coil, the antenna generates its own magnetic field and senses the existence of ferrous and non-ferrous material. The analogue output of the controller is then digitized and plotted on the computer screen. By taking advantage of this capability, the technology was used to retrofit backhoe excavators, trenchers, and augers. The success of the initially crude system led to the development of an improved version to be used during the excavation of unexploded ordinances (UXOs). In 1997, the Buried Utility Detection System (BUDS) consortium was founded that continued the work on three fronts: a) processing of sensory data, b) mechanical system, and c) human-machine interface. A simple user interface was created by creating a control box for the operator, consisting of two buttons for operating the articulated sensory platform and a red-yellow-green light as feedback. Details of the system and current developments, experiments and preliminary results will be discussed later. 
Utilities, such as gas lines and optical communication cables, are mainly nonmetallic making the EMI technology useless since these utilities are "invisible" to the metal detector. Other non-metallic objects underground include sewer lines made of concrete, clay and plastic. One sensor that has been successfully used even in archeology is the Ground Penetrating Radar GPR). The GPR transmits RF signals and detects the signals reflected by changes in the ground. When translated on the surface it provides a cross-sectional image of the material below the ground surface. Details of how this image is decoded, problems faced and implementation details are discussed later.

\section{THE NEED FOR DAMAGE PREVENTION}

The congressional Transportation Equity Act for the $21^{\text {st }}$ Century, TEA 21, Title VII, Subtitle C, SEC. 87301, states that: “... unintentional damage to underground facilities during excavation is a significant cause of disruptions in telecommunications, water supply, electric power, and other vital public services, such as hospital and air traffic control operations, and is a leading cause of natural gas and hazardous liquid pipeline accidents."

Underground Focus Magazine (1999) is a source that publishes an Accident File in every issue. For example it listed that from December $8^{\text {th }}$ until December $11^{\text {th }} 1998$, seven major accidents occurred. On the $9^{\text {th }}$ a fiber optic cable was cut by an excavation contractor that supported the 911 service for five counties in Jacksonville, Texas. The most tragic accident, however, occurred on Dec. 11 when "a crew using an "anchor cranker" to install a guy wire anchor for a telecommunications pole augured into a gas main." four people were killed and fourteen injured when the gas exploded in St. Cloud, MN.
There are many different parties, actively and passively, involved in the excavation and trenching process. Active participants include 1) owners of a new facility, 2) designers, 3) planners, 4) contractors, 5) utilities, 6) locators, 7) construction workers, and 8) equipment operators. In most U.S states, a contractor is required by law to call a "One-Call Center" 48 or more hours before he digs.

Despite the successful implementation of the One-Call systems in most of the U.S states, the accidents caused by damaging underground utilities resulting in wide variety of impacts reaching from a clogged residential sewer line to a gas explosion causing death and destruction. The list of impacted parties that incur cost comprises not only the contractor, utility and property owners, people in the vicinity of the accidents, but also the customers of a disrupted utility. Some of these groups include: a) private homes, b) governmental agencies, c) service companies, d) schools, e) hospitals, f) industrial firms, g) transportation systems like airports, taxi services, freight trains and trucking, $h$ ) retailers, and i) the utilities themselves. Overall, the direct and indirect costs of such accidents are staggering making the use of more sophisticated prevention approaches also economically prudent.

\section{LOCATING BURIED METALLIC UTILITIES USING EMI}

\subsection{CONCEPT AND BACKGROUND}

Anything metallic present in the ground can be induced to create a magnetic field, which can be detected by an antenna. The magnetic field is caused by a signal emitted by the transmitter coil. A receiver coil "listens" to this reflected signal and gets a measure of the metal around it. This can be done by either Continuous wave EMI or Pulse EMI.

EMI sensors have been used since the 1950 's for quality control on manufacturing production lines to safeguard against 
contamination. In more recent times, they have been used as a tool for mining, nondestructive testing, security, archaeology geology and other related fields. Metal detectors using electro-magnetic induction, especially pulse induction are not new in the field of buried utility detection. With two antennae, or one moving antenna in many positions, it is also possible to determine the depth of the buried pipe. Some researchers (Das et al., 1990) performed the analysis of the EMI detector for real-time location of buried objects. Several response characteristics of the utility, such as object depth, orientation, aspect ratio, and material properties were studied and, due to limitations of direct metal detection, the need for sophisticated processing was observed. This technology has also been used to discover unexploded ordinance (Lorenc and Bernold, 1997).

\subsection{A BURIED UTILITY DETECTION SYSTEM}

Figure 1 presents the basic idea of the equipment-mounted Buried Utility Detection System (BUDS) developed at NC State University. It is a prototype aimed at being part of a real-time system, integrating sensor fusion techniques. Presently, efforts are being made to form an extensive database of utility contours obtained from BUDS. After forming such a database, the next step is to go in for field-testing of unknown soils and utilities buried underneath. With a knowledge base and classification based learning schemes, we would be in a position to estimate with a degree of probability, the depth, orientation and material properties of the object. This estimation or the data obtained in the cruder stage of the sensing process would be fed into the sensor fusion module.

\subsection{EXPERIMENTAL TEST BED}

The experimental setup developed at CARL, called BUDS, consists of a moving cart under a stationary antenna. The cart and the antenna can be positioned at various angles to represent real-life site configurations. It is completely software controlled and is being used for collecting sensor contours for various geometric configurations of pipes, changing the material properties of the pipes and the antenna characteristics itself.

\subsection{INITIAL RESULTS}

Initial results (Fig 2) from the experiments show predictable agreement with previous work in the field. It has been found that as the antenna scans the utility below and the computer continuously plots the readings, a peak in the graph shows strong metallic content very near the antenna. By changing the horizontal angle and vertical tilt we get slightly different curves and by triangulation we can estimate depth of the pipe. We also noticed the change in the curves for deriving material characteristics, for example, studying the difference in the contours for a solid pipe and hollow pipe of the same dimensions and material.

\subsection{APPLICATIONS}

Apart from just carrying out experiments for detecting utilities, the CARL team has also worked on a BUDS application. It consists of the antenna mounted on a backhoe giving feedback to the operator about the existence of buried utilities before excavating (Fig 3).

\section{SUBSURFACE UTILITY MAPPING USING GPR}

\subsection{WHAT IS A GPR?}

The GPR is a remote sensing short-range system, which measures short pulse electromagnetic (EM) reflections due to variations of the electrical properties of the investigated medium. The electromagnetic wave, which is radiated from a transmitting antenna, travels through the material at a velocity that is related to the electrical properties of the material. As the wave propagates, if it hits an object or a boundary with different electrical properties, then part of the wave energy is reflected or scattered 
back to the source. The wave, that is reflected back, is captured by an antenna and an image is created that is reflective of the materials and boundaries present beneath the surface. The main drawback of a GPR is the inability to detect the exact material of the buried object.

\subsection{GPR USED IN PIPE AND MINE DETECTION}

New and general methods for landmine detection using GPR images have been evaluated. Simple and effective observation vector representations have been constructed to model the time varying signatures produced by the interaction of the GPR and the landmines. Gader (et al., 2001) used Hidden Markov Models (HMM) to recognize patterns and to predict the presence of landmines. Landmines appear in time domain GPR as shapes similar to hyperbolas, although corrupted by noise and other factors. A signature library was created using a combination of ground truth and GPR response for that truth-value. HMMs were used to generate probabilities for the unknown images by comparing them with the signature library present. The GPR has also been used for pipe detection. The same concept of creating signature libraries for different pipes at different depths is used and searching algorithms are used to predict the occurrence of pipes from unknown images. The image consists of distinct patterns, e.g. a hyperbola, which are studied to obtain a result.

\subsection{LABORATORY SETUP}

Most GPR image processing algorithms are based on a signature database that maps the different possible objects with their orientations and the images created by these objects under different soil depths and conditions. The optimal algorithm processes the image of the unknown object and compares it with those present in the database and generates an approximate estimation about the nature of the object. To study the responses of different pipe materials and other objects that could be present underground an experimental workspace (Fig 4) has been setup.

Most sample tests performed in the detection of mines or pipes, involved moving the GPR in one line, either forward or backwards. However, our setup aims at observing images in a single plane initially, and evolving into one that considers the perpendicular movement of the GPR. This would result in an overall zigzag movement that would generate a 3-D GPR image.

\subsection{CURRENT WORK / RESULTS}

The setup shown in Figure 4 is being used to study patterns generated by pipes of different materials, kept at various depths and also patterns generated by objects such as rocks and wood that might be present. The responses from various materials have been collected and the images are being studied to locate patterns unique to each object. The next stages include studying patterns generated by the zigzag motion of the GPR and creating a 3-D image that could be easily interpreted. Once the pattern recognition for pipe like structures has been studied, the next stage would be mounting the GPR with the EMI and obtaining a better estimation of the presence or absence of buried pipes. A sample GPR image of a buried pipe is shown in Figure 5.

\section{INCREASING EFFICACY OF BUDS USING MULTI-SENSOR APPROACH}

As mentioned above, the aim is to create a real-time, accurate, and most importantly reliable fish-finder type utility detection system with minimal false positives. Although the two sensors discussed above have their advantages, alone neither can give reliable estimation of the buried utilities. For example, the GPR module is reasonably accurate on the depth of the object, but is unable to distinguish between a metallic and a plastic pipe. Similarly, the EMI, by itself is not capable of providing accurate information regarding the position (e.g. 
depth) of a metallic object. These limitations of the individual sensors can be overcome by a fusion of sensor data.

According to Klein (1993), data fusion is a multilevel, multifaceted process dealing with the automatic detection, association, correlation, estimation, and combination of data and information from multiple sources. The type of fusion architecture used to combine sensor data depends on the application. There are three broad 'levels' where data fusion can be incorporated: a) direct fusion of sensor data, b) feature vectors and c) high-level inferences. Since the multi-sensor data in our case is not commensurate, we can either represent data obtained from each sensor via feature vectors, with their subsequent fusion; or perform individual processing of each sensor's data to achieve independent highlevel inferences or decisions, which are combined to make a collective decision.

The overall process consists of four main parts: Preprocessing the signal from the sensor, feature/contour extraction, feature/property selection, and classification. Figure 6 shows the adapted version of a common data fusion model to MS-BUDS (Multi-Sensory Buried Utility Detection System). The data from the two sensors are initially conditioned by independent signal processing modules which later feed into a parallel processor running a multisensor algorithm. The parallel processor uses a feature recognition and classification algorithm that operates on sensory data and 'learns' with a knowledge base as it goes along.

\section{SUMMARY}

Damage to buried utilities can cost lives and damage to property and equipment. This paper presented a novel technology that integrates two common sensory equipment, the Pulse EMI and GPR, into a multisensory real time underground utility detection system. The premise of fusing the two sensory data stream is to maximize reliability/accuracy while minimizing false positives. Two experimental facilities have been built to study the effect of various soil and object conditions on the features of the sensory outputs. In the next phase two experimental facilities will be combined into one platform, the MS-BUDS.

\section{ACKNOWLEDGEMENTS}

Funding for the work has been provided by Public Health Service-National Institutes of Health under contract 1R01 0H04201-01.

\section{REFERENCES}

Das, Y., McFee, J., Toews J., Stuart G.: "Analysis of an Electromagnetic Induction Detector for Real-Time Location of Buried Objects", IEEE Transactions on GeoScience and Remote Sensing v.28 No.3, May 1990.

Gader, P., Mystkowski, M., Zhao, Y.: "Landmine detection with GPR using Hidden Markov Models", IEEE Transactions on GeoScience and Remote Sensing v. 39 No. 6, 2001.

Klein, L.: "Sensor and Data Fusion Concepts and Applications", v. TT14, SPIE Press, 1993.

Lorenc, S.J., Bernold, L.E: "Excavatormounted Ordnance Locating System for Remote Excavation", 14 ${ }^{\text {th }}$ International Symposium on Automation in Construction, June 1997.

Underground Focus Magazine, (1999). Canterbury Communications, Spooner, WI. URL: http://www.underspace.com/ 


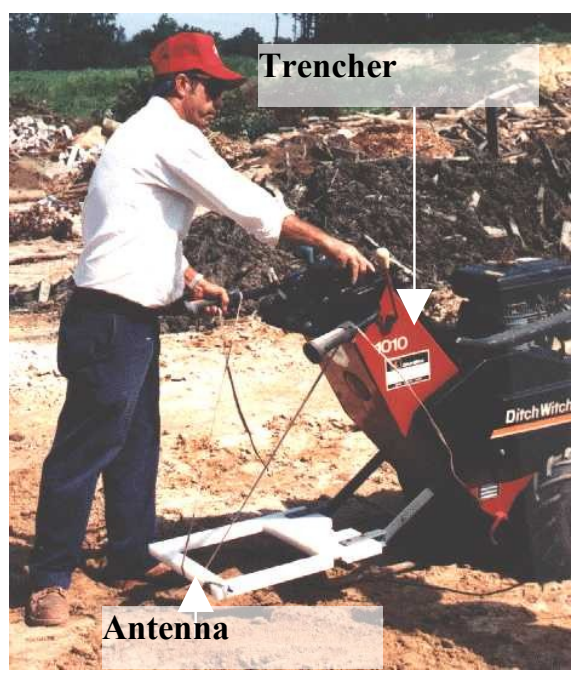

Fig. 1. Equipment mounted BUDS

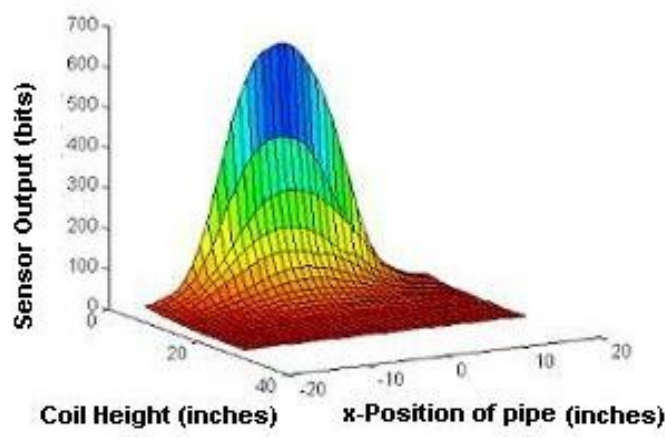

Fig 2. Graphical sensory output of BUDS

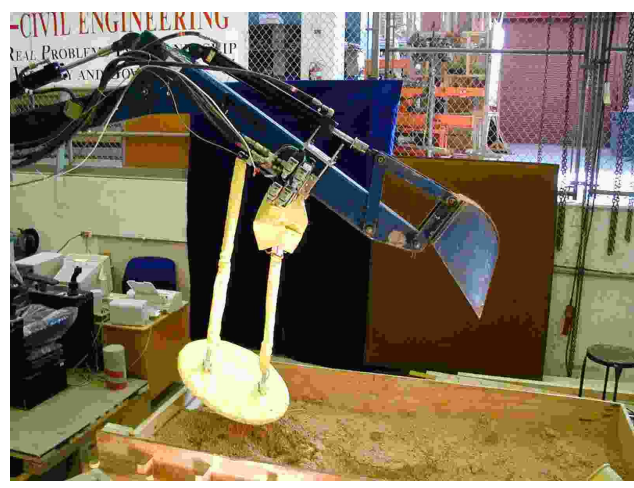

Fig 3. Buried Utility Detection System mounted on a backhoe.

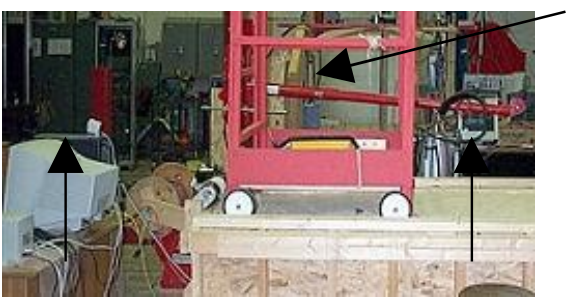

Image Processor

Sandbox

Fig 4. GPR setup for utility detection.

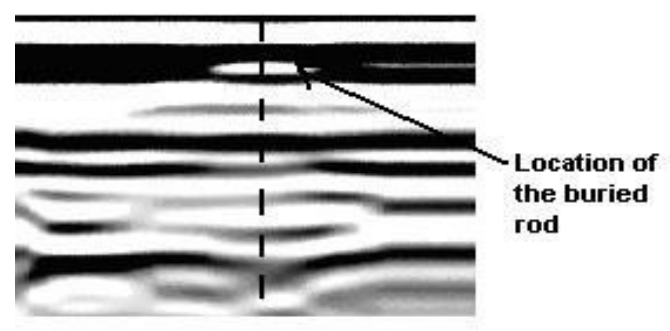

Fig 5. Sample GPR image of a buried rod

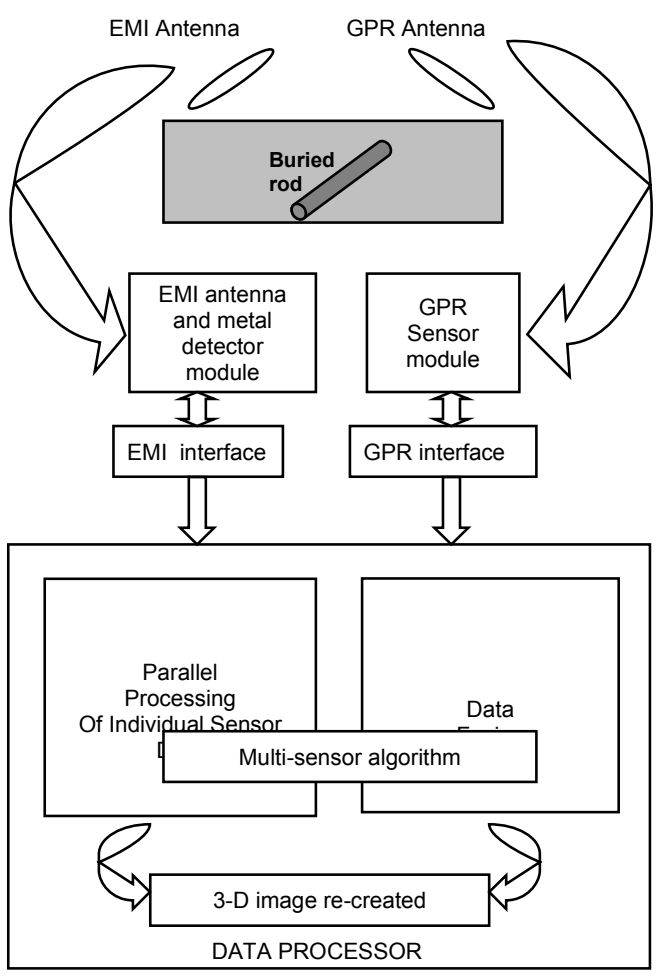

Fig 6. Simplified data fusion model adapted to MS-BUDS 